\title{
BMJ Open Quality A team approach to the introduction of safe early mobilisation in an adult critical care unit
}

\author{
Sanjiv Chohan, Sara Ash, Lorraine Senior
}

To cite: Chohan S, Ash S, Senior L. A team approach to the introduction of safe early mobilisation in an adult critical care unit. BMJ Open Quality 2018;7:e000339. doi:10.1136/ bmjoq-2018-000339

- Additional material is published online only. To view please visit the journal online(http://dx.doi.org/10.1136/ bmjoq-2018-000339).

Received 22 January 2018 Revised 13 August 2018 Accepted 2 October 2018
(A) Check for updates

(c) Author(s) (or their employer(s)) 2018. Re-use permitted under CC BY-NC. No commercial re-use. See rights and permissions. Published by BMJ.

Department of Intensive Care, Monklands Hospital, Airdrie, North Lanarkshire, UK

Correspondence to Dr Sanjiv Chohan, Department of Intensive Care, Monklands Hospital, Airdrie, North

Lanarkshire, UK;

sanjivchohan@nhs.net

\begin{abstract}
Delirium and intensive care unit acquired weakness are common in patients requiring critical care and associated with higher mortality and poor long-term outcomes. Early mobilisation has been shown to reduce the duration of both conditions and is recommended as part of a strategy of rehabilitation of critically ill patients starting during their stay in intensive care. Our aim was to achieve 95\% reliability with a standardised mobilisation process. Multidisciplinary involvement through the use of regular focus groups lead to the development of a standardised process of sitting a ventilated or non-ventilated patient at the side of the bed for a set period of time, which was called the daily dangle. Team learning from Plan, Do, Study, Act (PDSA)cycles, as well as feedback from both staff and patients, allowed us to develop the process and achieve a median $87 \%$ reliability. Delirium rates fell from $54.1 \%$ to $28.8 \%$. There was no change in average length of stay, and no adverse events. Ownership by the staff, development of the process by staff, iterative testing and learning, and designs for reliability were the factors behind the successful adoption of a new and challenging process. Particular changes which drove reliability were standardisation of the criteria for a dangle, standardisation of the dangle itself and a reminder included on the daily goals checklist.
\end{abstract}

\section{AIM}

The aim of the project was that $95 \%$ of patients who met standardised criteria in the intensive care unit (ICU) would be mobilised according to a standardised process by December 2016.

\section{Problem}

This project was carried out in an adult ICU located in a hospital in an urban area just outside Glasgow. The unit had six beds and looked after only level 3 patients, defined as 'patients receiving advanced respiratory support alone, or a minimum of 2 organ supports'. ${ }^{1}$ The unit admitted between 300 and 350 patients per year, and was located in a district general hospital with a busy emergency department and a range of acute surgical and medical specialties. The majority of the patients was intubated and ventilated (more than $90 \%$ - data from the national database Ward
Watcher). In April 2016, the unit relocated to a new facility in the same building which now takes level 2 (patients needing extended postoperative care or single organ support ${ }^{1-}$ high dependency unit (HDU)) and level 3 patients, and has expanded its capacity to 10 beds, taking approximately 650 admissions per year. It is staffed full time by nursing and medical staff, and Monday to Friday by dedicated physiotherapists, with on-call physiotherapy staff at weekends. The unit had achieved reliability (greater than $95 \%$ of patient days) of daily interruption of sedation as part of the Scottish Patient Safety Programme by the end of 2012. The standard sedation/analgesia medications in our ICU are propofol with an opioid, usually morphine or alfentanil. Benzodiazepines are rarely used. ICU acquired weakness (ICUAW) is common in critically ill patients and is associated with poor outcomes. The duration and severity of this condition can be reduced by the early mobilisation of patients. We did not have the resources to measure the incidence, duration and recovery from ICUAW in our patient population, but it was regularly diagnosed during ward rounds. Delirium is a common complication of critical illness associated with poor patient outcomes. Daily screening for delirium was introduced in the ICU in March 2013 using the confusion assessment method for the ICU (CAM-ICU) delirium screening tool, a validated tool for use in intensive care. ${ }^{2}$ Between April and August 2014, a baseline measurement of delirium rates found that approximately $60 \%$ of critical care patients were delirious at the time of measurement. All patients had multiple recognised risk factors for the delirium present. Staff focus groups were formed to try to identify which of the risk factors for delirium we believed were present in our patients, and that we would want to reduce or eliminate. Noise, disturbed sleep and mobilisation were identified as areas where we could improve processes. A number of measures to reduce noise levels were introduced such as reducing the number of machine alarms, and 
efforts to improve sleep including the use of ear plugs and eye masks, but these did not affect delirium rates. We therefore decided to introduce processes to ensure that patients were mobilised as early as possible as a way to potentially reduce delirium rates, and to reduce the duration and severity of ICUAW. We were aware of work in other ICUs that had introduced mobilisation processes including sitting patients at the edge of the bed, and walking, both very challenging while intubated and ventilated. These processes were not part of the normal work routine in our ICU, although they had both occurred on a few occasions when agreed by the clinical team.

\section{Background}

Delirium is an acute, fluctuating syndrome of inattention, impaired level of consciousness and disturbed cognition (National Institute for Health and Care Excellence (NICE) ). ${ }^{3}$ Delirium is a non-specific but generally reversible manifestation of acute illness. ${ }^{4}$ It is classified in three subgroups; hypoactive, hyperactive and mixed delirium. Delirium incidence in the ICU varies between $16 \%$ and $89 \% \cdot{ }^{56}$ NICE guidelines, as well as published critical care guidelines on the management of pain agitation and delirium recommend daily screening of patients for delirium and treatment of underlying causes. ${ }^{37}$ There are multiple risk factors for delirium including sedation, hypertension, alcoholism, severity of illness, age and infection, with many patients having more than one risk factor, ${ }^{89}$ although the exact mechanism of its development remains unclear. Delirium is associated with increased risk of death, decreased long-term cognitive function, and increased length of hospital stay. ${ }^{10-14}$

ICUAW is the name given to the consequence of acute illness that may include a neuropathy, myopathy or some combination of the two. ${ }^{15}$ It is defined as 'a clinically detected weakness in critically ill patients in whom there is no plausible aetiology other than critical illness'. ${ }^{15}$ The incidence of ICUAW has been estimated between 25\% and $100 \%$ with multiple risk factors including sepsis, the use of neuromuscular blocking agents, hyperglycaemia and physical inactivity. ${ }^{1617}$ Functional disability can persist for years after recovery from the acute illness. ${ }^{18}$

Early mobilisation and physiotherapy has received increasing attention as a process which may improve these outcomes. A trial of physiotherapy and occupational therapy within 72 hours of intubation and ventilation showed that patients in the intervention group had half the number of days with delirium than those who received usual care. ${ }^{19}$ A quality improvement project which focused on reducing deep sedation and mobilising patients early in their ICU stay showed a reduction in the number of patient days with delirium and coma. ${ }^{20}$ In both studies, it is unclear if the effect on delirium was due to mobilisation or to intended or unintended change in sedative use. A strong possibility raised by these studies is that introduction of early mobilisation itself prompted a change in sedation practice.
A systematic review of active mobilisation of patients who were ventilated for more than 24 hours found that active mobilisation may improve muscle strength, functional independence, weaning from the ventilator, and reduce hospital length of stay. ${ }^{21}$ No serious adverse events were reported in the studies reviewed. A more recent systematic review which included studies of active therapies including sitting at the edge of the bed, and active exercises, hoisting to a chair, standing and walking, found that active mobilisation led to better muscle strength at ICU discharge, greater probability of walking without assistance at hospital discharge, and more days alive and out of hospital at 180 days. It found no difference in ICU or hospital length of stay, quality of life, or duration of mechanical ventilation. ${ }^{22} \mathrm{~A}$ randomised control trial of goal-directed mobilisation examined the effect of graded mobilisation in ICU patients. Patients who were functionally independent prior to ICU admission, and who were ventilated for less than 48 hours during their ICU stay, were mobilised according to goals set on ward rounds, either no mobilisation, passive movements, sitting, standing or walking. Interprofessional planning and working was assured by a facilitator who ensured the delivery of the mobilisation protocol. The intervention led to higher mobilisation scores, shorter length of stay and greater functional mobility at hospital discharge. ${ }^{23}$ A pilot study randomised patients expected to be ventilated for more than 48 hours to usual physiotherapy or early goal-directed mobilisation. This was a protocolised graded intervention consisting of 30-60 min of activity consisting of sitting in bed, sitting at the edge of the bed, sitting in a chair, standing or walking, depending on the mobility assessed by a scoring system. The study found that this approach was feasible and safe, and improved mobilisation rates with double the proportion of patients in the intervention group walking. Although not powered to look at other outcomes, there was no difference in ICU or hospital length of stay or duration of ventilation. ${ }^{24}$

Results of trials so far have not been consistent in showing improved outcomes although the research so far has led to widespread attempts to adopt early mobilisation in ICU. NICE guidelines in 2009, subsequently updated in 2017, recommend that rehabilitation start as early as possible during a patient's stay as part of a structured rehabilitation programme. ${ }^{25}$

Questions remain on the optimal patient group, which outcomes are affected and the optimal mobilisation process.

Thus far, mobilisation of ICU patients has been found to be safe, with events with any real patient consequence being rare, although there is heterogeneity in the definition of safety events in different studies. ${ }^{26}$

There are a number of barriers to mobilisation. A 2015 study found that in Scottish ICUs, $40.1 \%$ of ventilated patients were mobilised. The study examined barriers to mobilisation, and found that sedation, an endotracheal tube in place and cardiovascular instability were the most common. ${ }^{27} \mathrm{~A}$ systematic review examining the 
barriers to mobilisation looked at qualitative and quantitative studies, predominantly focused on mobilisation in the ICU as opposed to the post-ICU setting, and found that patient physical and psychological factors, safety concerns, culture, leadership, motivation, beliefs regarding risks, and environment all acted as barriers. The majority is modifiable and could be addressed in a programme aimed at introducing early mobilisation. ${ }^{28}$ Another systematic review found that patient, clinician, protocol related and ICU contextual factors all have an influence on the adoption of early mobilisation. ${ }^{29}$ A number of the studies cited used designs to address barriers to mobilisation, such as mobilisation teams, goal setting on ward rounds, criteria for mobilisation, interprofessional working and mobilisation protocols.

\section{Measurement}

The primary purpose of this project was to introduce a new process and so reliability with the mobilisation process was our primary measure. We decided to measure average length of stay and delirium rates as outcome measures, although both outcomes are affected by multiple factors, not just mobilisation. However, both could potentially be affected by mobilisation, and were established measures in ICU. We chose average length of stay as the primary outcome measure based on the evidence of the impact of ICUAW and the potential of early mobilisation to improve muscle weakness. Data were extracted from the national ICU database (Wardwatcher) and calculated by dividing the total number of ICU patient days per calendar month divided by the number of patients discharged from the ICU that month, a standard metric in Scottish ICUs. ${ }^{30}$ The baseline average length of stay was 3.5 days.

Daily screening for delirium was introduced in March 2013 prior to the start of this project, in line with guidelines. Initially screening for delirium was sporadic. ${ }^{31}$ A project team of nurse practitioner, a junior doctor and ICU consultant designed a multifaceted teaching package on ICU delirium and CAM-ICU. This comprised an introductory poster relating a patient's experience of ICU delirium, a slide show presentation, desktop information leaflet outlining the impact of ICU delirium, bedside demonstrations, supervised performance of the CAM-ICU and provision of a pocket card to aid continued delirium screening. Nursing staff were surveyed on their attitudes to and knowledge of ICU delirium before and after teaching. Spot checks were also undertaken to confirm competency in performing the CAM-ICU postintervention. The project team continued to monitor the recording of the presence or absence of delirium, and the accuracy of screening. Feedback to staff on the results continued. Screening was found to be reliable and accurate in early 2014. Weekly data were collected on random days by the project team. The accuracy of screening was checked at the time of data collection. Random days for data collection were identified using a random number generator on Microsoft Excel. On those days, all patients were included in the initial sample, with patients being then excluded if they did not meet criteria for delirium screening. The numbers of patients with and without delirium were recorded, and rates calculated by dividing the number of patients with delirium by the total number of patients eligible for delirium screening. The baseline median rate was $60 \%$. As the project progressed, data were aggregated and rates calculated for each calendar month.

Process data on mobilisation rates were collected one random day per week. Data were collected on:

- Number of patients in ICU at that time.

- Number of patients who met the criteria for three mobilisation processes-the dangle, the stand and sitting in a chair (see next section for operational definition) before 10:00.

- The number of patients who mobilised according to a standardised process (see below for operational definition) before 17:00 when data were collected.

All data were collected by a core team of three staff members which ensured validity and reliability of measurement. Data were plotted as a time series to make run charts, initially weekly (sample size 1-3), and later aggregated to give monthly data (sample size 5-10). The sample size approximately doubled from April 2016 when the unit moved from a 6 -bedded facility to a 10-bedded facility. Outcome data were plotted on statistical process control charts later in the project.

As a balancing measure, we counted the number of adverse events, such as dislodgement of endotracheal tubes, or central lines that occurred during mobilisation. In the early part of the project, staff were initially concerned that mobilisation sometimes seemed to leave patients exhausted and may have 'set them back'. We therefore also measured average length of stay on a ventilator as a balancing measure. This is defined as the total number of ventilator days provided by the ICU each month, divided by the total number of patients who were ventilated during that month.

Feedback from patients and staff was collected in the form of quotes and presented at focus groups for discussion.

\section{Design}

A core project team of an advanced nurse practitioner, ICU consultant and physiotherapist was formed. We began by examining mobilisation practice in other ICUs, and seeking advice on how they went about introducing the process. In addition, one of the team members (author SC) had visited ICUs in Inter Mountain Health, Salt Lake City and seen their work on mobilisation. This group has particular experience and knowledge of mobilisation. ${ }^{32}$ This group had adopted the nomenclature 'The daily dangle' for the process of sitting a ventilated patient at the edge of the bed for a set period of time, and this name was unofficially adopted by our unit. The group also reviewed the published literature to help plan the project. Roger's theory on the diffusion of innovations, which examines factors affecting the adoption of new 


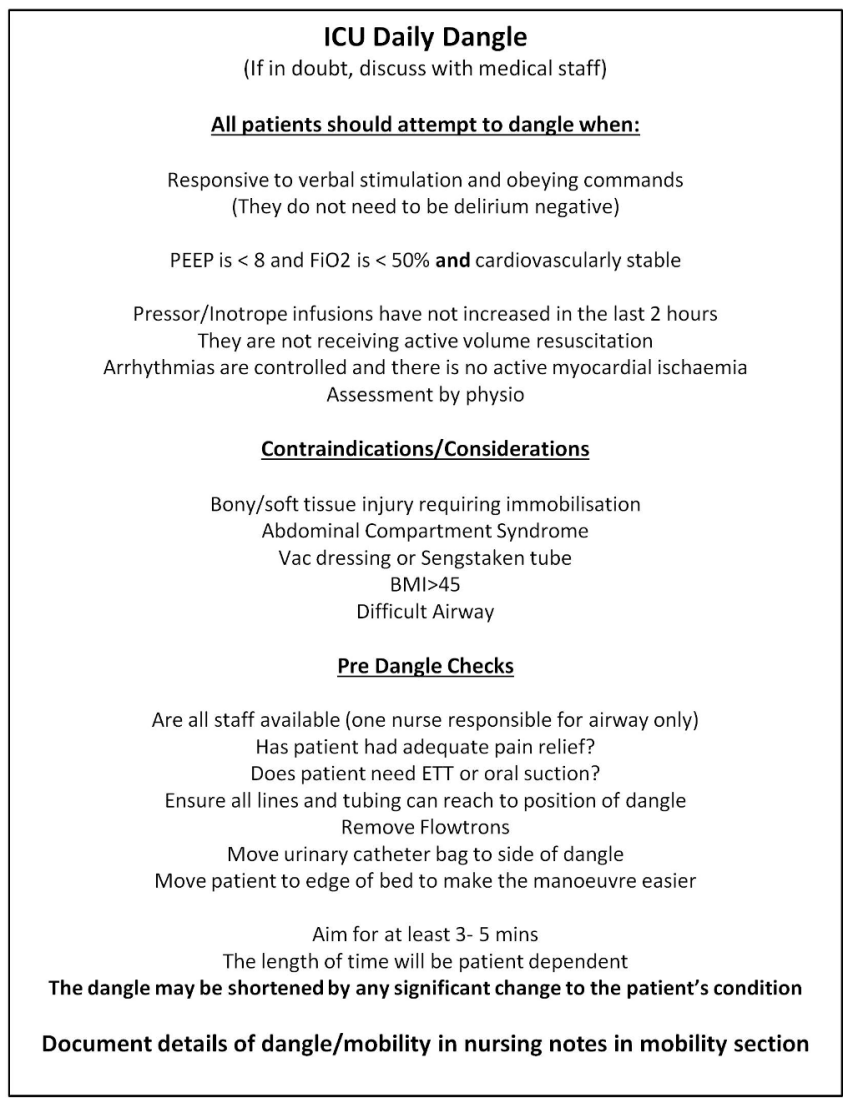

Figure 1 Initial protocol for daily dangle. BMI, body mass index; ICU, intensive care unit; ETT, endotracheal tube; $\mathrm{FiO}_{2}$, inspired oxygen fraction; PEEP, positive end expiratory pressure.

technology or processes, suggests that some people will be more willing than others to try a new process, and that its further adoption will be dependent on, among other factors, the ability to test it, observe its effects and adapt it for local needs. ${ }^{33}$ A Health Foundation evidence scan examined the barriers to successful quality improvement in the National Health Service (NHS), and found many factors, including lack of ownership by professionals and their lack of autonomy to make changes to the process. ${ }^{34}$ The project team understood that the mobilisation process would be delivered and experienced by nursing and physiotherapy staff, and by patients. We therefore decided to ask the staff to design and test the changes with feedback from patients and families. We instituted weekly staff focus groups, led by one of the project team, within the clinical area, thus minimising disruption to clinical care, of about 10-15 min each, where the results of tests of change could be discussed and new tests agreed. Frequent focus groups ensured the input of broad groups of staff. At the first set of focus groups, the background to the project and the supporting evidence were shared with the staff and their views solicited. Although staff expressed a number of reservations around the difficulty of mobilising ventilated patients, and the risk and benefits, agreement was reached to test changes cautiously. The staff identified four mobilisation processes: sitting a patient at the edge of the bed ('the daily dangle'), sitting a patient in a chair, standing up beside their bed and walking. The dangle was chosen as the first area to focus on. Nursing staff wanted the involvement of physiotherapists in the process of mobilising patients, at least initially, to provide some expert guidance on an unfamiliar process, which was arranged. In the next set of focus groups, staff chose the patient selection criteria for a dangle to be modified though testing. Thereafter, they defined the process of a dangle, again with the expectation of modifying this through testing. Tests of change were carried out using Plan, Do, Study, Act (PDSA) methodology. Results were shared at focus groups. Data, as well as feedback from patients and staff in the form of word clouds and free text, were posted on the quality improvement board in the ICU.

\section{Strategy and improvement cycles}

Our tests of change using PDSA cycles are described below. Some of these contain a number of PDSA cycles but have been grouped together. Our aim was that $95 \%$ of patients, who met the criteria for a dangle, would be mobilised according to the dangle process by December 2016. Our strategy was first to define the eligible patient group, then to standardise the dangle process, and then to test a series of reminders to improve reliability.

\section{PDSA 1}

Staff agreed on a set of written criteria to select a patient for the first dangle, and a process to be followed. Nursing and physiotherapy staff would jointly carry out the first dangle on a single patient. Our hypothesis was that we could sit a ventilated patient at the edge of the bed without dislodging invasive devices, or ventilatory or haemodynamic deterioration. The initial patient selection was very cautious. The patient was able to obey commands, was intubated, breathing $30 \%$ oxygen, on a spontaneous mode of ventilation with minimal support, receiving no inotropic support. Three nursing staff and one physiotherapist were employed in the process who discussed the process beforehand, with one to support the ventilator tubes, and the physiotherapist to support the back. The patient was sat at the edge of the bed for 3 min after which his head began to droop. Staff reported no adverse events. They also reported that the patient unexpectedly seemed to become more alert and tried to support his own weight.

\section{PDSA 2}

After sharing the results of PDSA 1, we decided to repeat the above test with a series of individual patients, reviewing the results after each dangle to inform the next. Our hypothesis was that as staff gained confidence and experience with mobilising ventilated patients, the initially cautious criteria and process would develop to include a larger group of patients who might benefit. These were carried out over a number of weeks, with a variety of different staff but always with a physiotherapist, 


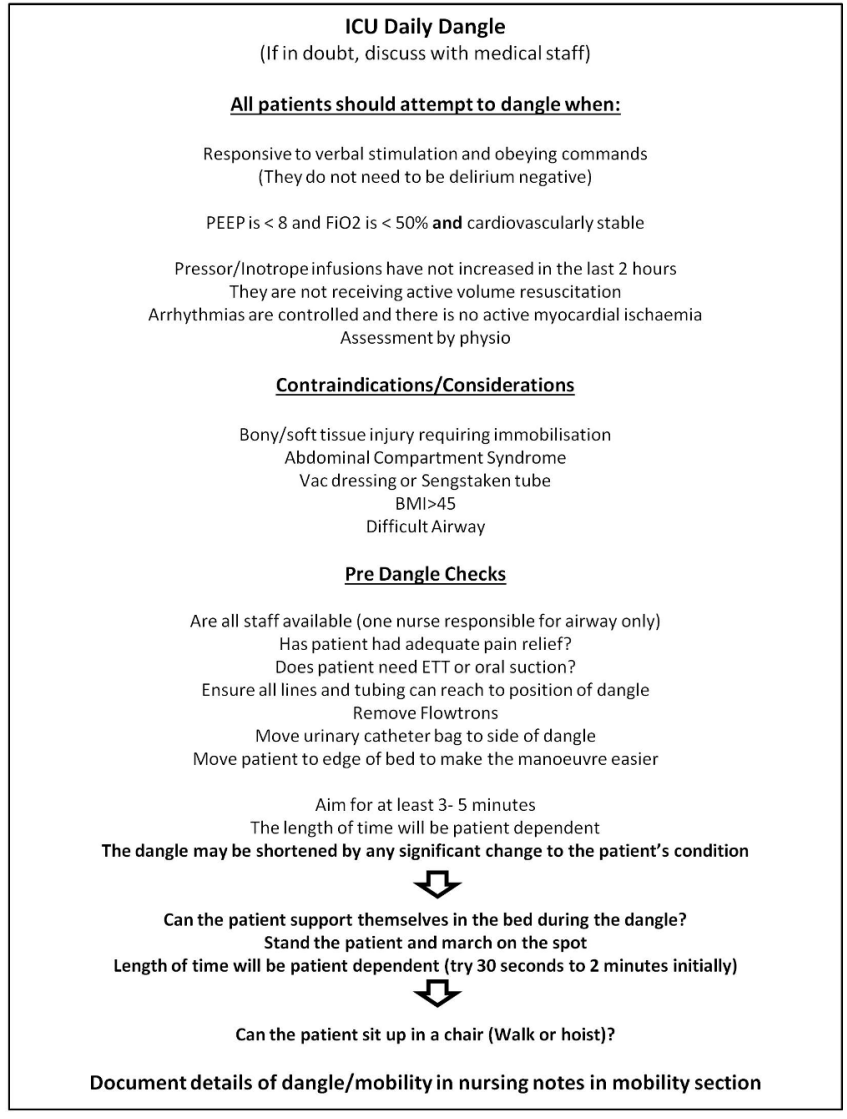

Figure 2 Revised protocol for daily dangle. BMI, body mass index; ETT, endotracheal tube; FiO2, inspired oxygen fraction; ICU, intensive care unit; PEEP, positive end expiratory pressure.

with no adverse events. As staff gained confidence, the team found that the staff had chosen to mobilise patients who did not strictly meet our initial criteria, but instead were requiring more physiological support than had been initially agreed on. The eligibility criteria were modified iteratively (figures 1 and 2). Staff believed the process to be easier than expected, to have sometimes led to the similar phenomenon of patients becoming more alert, to be comfortable for patients and to be safe. Teamwork with physiotherapists was welcome and enjoyable. Feedback from two patients had both (one after extubation and one in writing) reporting that they did not experience any adverse symptoms from the dangle. One patient had asked for more equipment and opportunity to exercise to help his recovery.

\section{PDSA 3}

Testing the dangle process on weekdays when physiotherapy support was available. Our aim was to deliver the dangle to the majority of patients who met the criteria each day, allow staff to build experience with the process, and to inform any further changes to the criteria or process itself. Our hypothesis was that the majority of staff had become aware that this process was being tested, had had a chance to discuss it, were comfortable with the process and could act as teachers to the others. Some had suggested it could ultimately be delivered by nurses alone without physiotherapists, but not at this stage. We predicted there would be some difficulty in delivering the dangle to every patient every day due to lack of familiarity, and lack of experience in co-coordinating this process with the other work of the day. Data collection on mobilisation rate commenced. Results: Over the next few weeks, a median of $52 \%$ of patients achieved the dangle. No adverse events were reported. Staff quickly became comfortable with the process, and found that often only two staff were needed. No changes were suggested to the eligibility criteria or the process itself. Some staff chose during weekdays to mobilise patients without the involvement of physiotherapists, and to mobilise at weekends despite not having a dedicated ICU physiotherapist. On a number of occasions, mobilisation was discussed on the ward rounds with medical staff, prior to a decision being made. There was variation in the decisions made, sometimes without referral to the criteria. It was more challenging to fit mobilisation into the work of the day on busy days.

\section{PDSA 4}

There is an established, reliable $(>95 \%)$ system of daily goal setting at our ICU ward rounds. We tested a prompt on mobilisation within the daily goals. Our hypothesis was that medical staff were less familiar than nursing and physiotherapy staff in the criteria for the dangle, and that some staff preferred to discuss mobilisation with the wider team prior to making the decision to perform the dangle. We predicted an increase in the number of patients achieving the dangle. The six ICU consultants were taught the local process and shown results so far. All were aware of the project and supported its aims, but did not know our specific process. Results: over the next 3 months we saw an increase in the number of patients achieving the dangle to a median of approximately $80 \%$, although still with day-to-day variation. A number of changes to the wording of the daily goals prompt were made. Staff did not suggest any further changes to the eligibility criteria, but did emphasise that sometimes the team used their judgement to mobilise patients who did not strictly meet the criteria. Feedback reported that some patients were now being mobilised to sitting out of bed in chairs after discussion on the ward round, and that the dangle process might be too limited a goal. No adverse events were reported. The main barrier to mobilisation was the challenge of fitting this new process into the work of the day, with evenings being most challenging, and staff stated that a chance to plan as a whole team might help.

\section{PDSA 5}

Planning mobilisation, reduced situation awareness over a day and lack of time were barriers. We therefore decided to test a ward brief, which staff called a safety brief in the early afternoon, to aid team planning. The nursing team would gather to determine which patients remained to be mobilised, the personnel required and how to deploy 
themselves. Results: over the next 3 months afternoon safety briefs occurred on about $50 \%$ of all days. When it occurred, it did allow planning, but remained person dependent. This reflected that an afternoon safety brief itself was an additional process and could be challenging to achieve. No improvements in mobilisation rates were seen. No adverse events were reported and feedback remained positive.

\section{PDSA 6}

From April 2016, the unit moved to a new facility, which now treated level 2 as well as level 3 patients with the merging of two nursing teams. The next few months were spent familiarising staff with new patient groups and with the processes of the new combined ICU/HDU. No specific changes were made to improve the rates at which patients received the dangle, but the focus groups were continued as a way of teaching the process to new staff. We predicted a decrease in mobilisation rates but no reduction was seen. A reduced variability in the dangle rates was seen as the sample size, which now included level 2 patients, increased. Feedback from the focus groups remained positive about the dangle, and many new staff who had previously worked in a surgical HDU were very supportive and became advocates for it. There was strong feedback that we should formalise other mobilisation processes more suitable for the level 2 group including sitting in a chair, standing and walking, but include the level 3 patients where suitable. Verbal feedback from patients was now more readily available as some were not intubated. Occasionally patients would report that mobilisation was tiring, but there were no reports of pain, and patients felt positive about moving. We found that explanations to the patients and families on the purpose and practice of mobilisation were readily understood and accepted.

\section{PDSA 7}

The next phase of testing was not specifically to improve reliability of the dangle, but to introduce further mobilisation processes-the stand, walk and sitting in a chair. The aim was to test standardised criteria and processes as we did for the dangle, and to achieve their reliable use, initially for $50 \%$ of patients. Weekly focus groups were used to agree, modify and test the processes. We collected data on the number of patients eligible for each process, and the number who achieved them. Results: staff saw these processes as continuations of the dangle, so that if a patient was able to dangle, then the staff would proceed to standing the patient at the side of the bed, marching on the spot, and then if still able, to sit out of bed in a chair. They did not refer to eligibility criteria for walking, standing or sitting. No minimum time was set for sitting in a chair, but when patients were sat in chairs, in practice it was for at least $30 \mathrm{~min}$ and could be for up to 3 hours according to patient preference or fatigue. After the first 4 months reliability with the processes rose to above $80 \%$ as more staff were taught the new processes and were able to become familiar with them. Staff believed the processes to be useful, achievable but sometimes challenging when busy. We saw less reliance on physiotherapists, and mobilisation was now practised at weekends routinely, sometimes with the involvement of on-call physiotherapists, sometimes by nursing staff alone. No adverse events were reported.

\section{PDSA 8}

New equipment was purchased using a donation to the ICU. Feedback from patients had showed an appetite to take part in exercise and mobilisation. Chairs were not always readily available to mobilise patients, and had to be borrowed from elsewhere in the hospital. Our hypothesis was that purpose-made chairs stored in the critical care unit would make it easier to sit patients out of bed at a time suitable for patients and staff. In addition, we bought a hand cycling machine and some free weights which could be used by patients under the guidance of physiotherapists. The pieces of equipment were not expected to make any impact on the rates of mobilisation, but are mentioned for completeness. Results: we saw no change in the rates on mobilisation, but feedback from staff was that they felt the patients were more comfortable than in old chairs, and having ready access allowed planning of the work day. There were no adverse events.

\section{PDSA 9}

From December to January 2016, we saw a decrease in rate of all mobilisation processes. Winter is an especially busy time for critical care. There was also a high number of staff off sick, leaving fewer available to cover shifts and leading to fatigue. Mobilisation required at least two members of staff, and in the busy period, it proved to be difficult to always mobilise patients. Decision making by medical staff was still variable, and staff seldom consulted written criteria. We therefore decided to test a decision making tool, to simplify the identification of eligible patients for mobilisation. Decision making tools such as checklists have been successfully used to reduce errors. We predicted that a tool would be easy to create, and would improve the reliability of mobilisation. Results: a tool in the form of a tick box sheet was produced, and tested for accuracy and ease of completion. It was modified until we found that 10 staff members were able to complete it easily and accurately for their patient. We then asked all members of nursing staff to use it in the morning prior to the ward round, to identify patients for 1 week. We found that it was frequently not filled in, and not often referred to on the ward rounds. Staff reported that it may be useful if completed jointly by medical and nursing staff on ward rounds. Despite this, mobilisation rates returned to baseline levels as staff numbers returned to normal. 


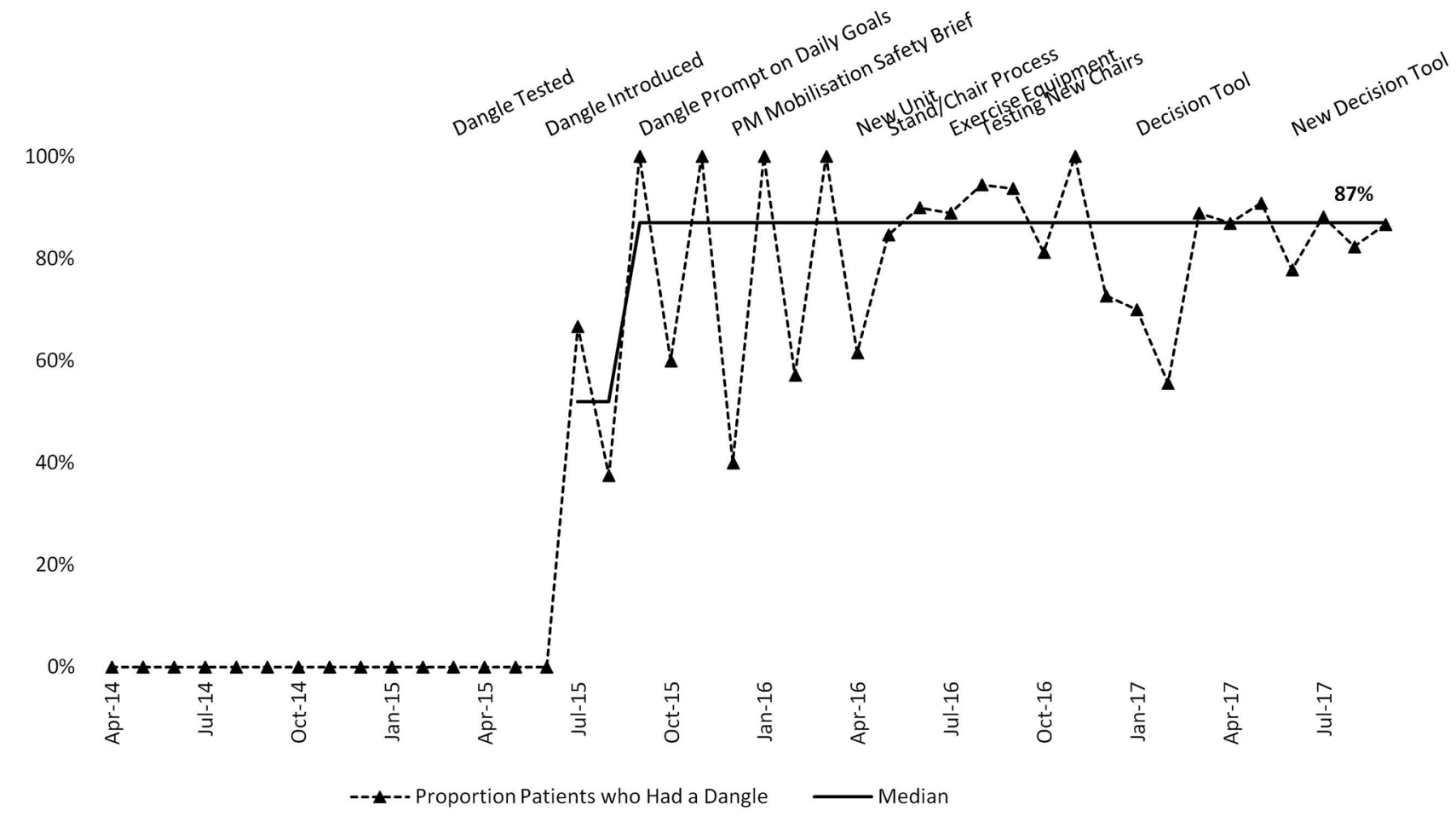

Figure 3 Proportion of patients who had a daily dangle. (PM - afternoon)

\section{RESULTS}

We achieved $87 \%$ reliability with the dangle process from a baseline of $0 \%$ (figure 3). The key interventions which drove this were: creating standardised criteria for the dangle, creating a standardised process for the dangle and including a prompt on the daily goals checklist. From April 2016, the unit moved from a 6-bedded stand-alone level 3 unit to a combined 10-bedded level 2 and level 3 unit. Reduced variability in the dangle rate was seen reflecting the larger number of patients in the sample. A decrease in the dangle rate was seen between December 2016 and April 2017 due to the increased workload. The rate returned to baseline after this period largely due to a renewed focus on the project but was coincidental with the introduction of the dangle decision making tool.

Average length of stay showed special cause variation in July 2017 using control chart rules, indicating a non-random change, followed by reduced month-tomonth variation (figure 4 ).

A median of $80 \%$ of all patients were eligible for assessment for delirium using the CAM-ICU (figure 5). Delirium rates as measured on a statistical process control chart fell from a mean of $54.1 \%$ to $28.8 \%$ as detected by a rule of 8 or more sequential data points below the baseline mean (figure 6). This occurred shortly after the dangle was introduced. A further decrease was seen after January 2017 as measured by nine sequential data points below the mean.

Balancing measures-there were no adverse events reported during this project. There was no change in the average length of stay on a ventilator during this project (figure 7).

\section{LESSONS AND LIMITATIONS}

This project successfully introduced a new process, the daily dangle, into a clinical service. The project design explicitly aimed to address some of the known barriers to early mobilisation. ${ }^{28}$ This process was a change in practice with which the staff group had no experience, and was a developing practice in ICU that was not yet established, although some of the project team had visited other ICUs where this practice had been introduced. In addition, it raised some anxiety in staff that the process carried risks to patients as it was perceived to be a radical change from current practice. The key factor behind its successful introduction was the ownership and control of the introduction by the staff that had to deliver the process. Encouraging the team to adapt what had been developed elsewhere to local conditions and needs has been widely reported as a successful strategy in quality improvement. Discussions through the use of short focus groups with staff in the ward area were a practical and effective way of engaging staff, planning and reviewing changes. This approach allowed frequent discussions with staff in a service where regular formal meetings are difficult to deliver. The use of staff and patient feedback within the focus groups was effective in building confidence in the process which drove development of the process from an initially very conservative one to one affecting a much larger group of patients. We found no adverse events, consistent with studies which have demonstrated a low rate of adverse events. Feedback of this data to staff was also important in building confidence. A limitation of our project was that we did not formally analyse feedback and so use it as a measure. As a driver of change, it 




Figure $4 \mathrm{U}$ chart average length of stay. (PM - afternoon)

was effective, and also drove a team approach by ensuring that staff felt listened to.

We achieved a median $87 \%$ reliability with the process, less than our aim of $95 \%$, but with periods of sustained reliability. The winter period of 2016 to 2017 showed a decrease in reliability indicating that in busy periods it was still difficult to deliver a labour intensive process. We incorporated designs for reliability ${ }^{28}{ }^{35}$ to ensure sustainable process reliability. Standardisation of the criteria for the dangle, and the dangle process itself helped in

- Proportion of all ICU patients who could be assessed for delirum Median

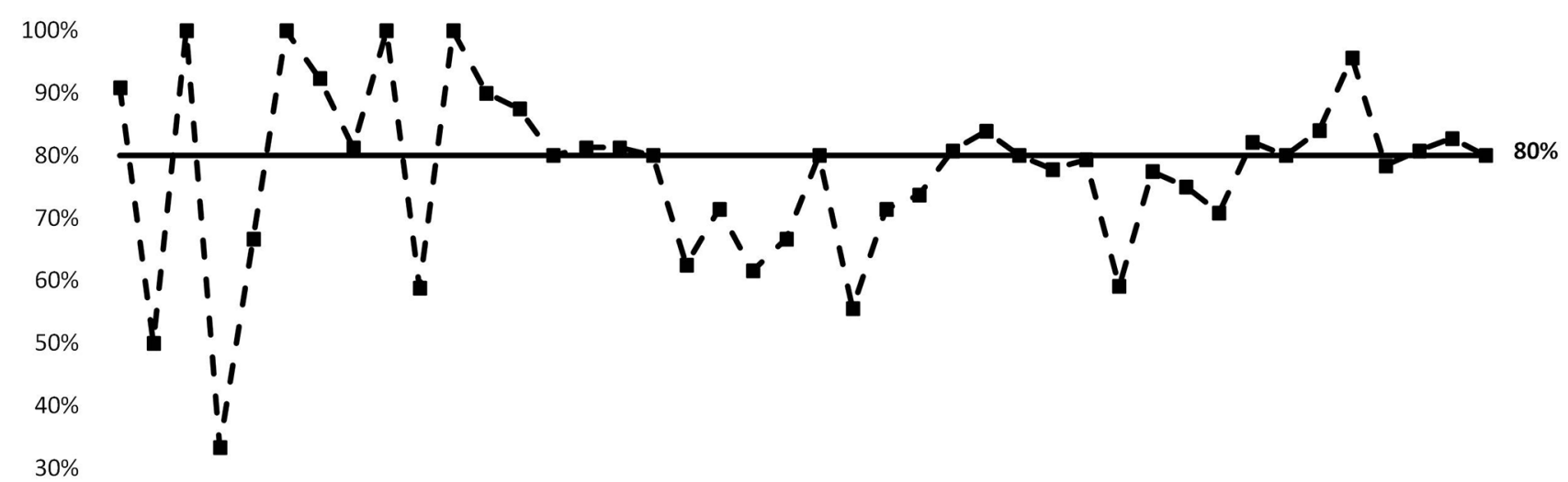

$20 \%$

$10 \%$

$0 \%$

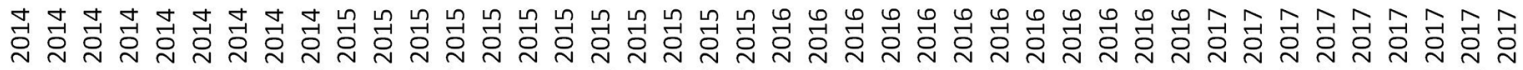



Figure 5 Proportion of all patients included in the delirium sample. ICU, intensive care unit. 




Figure 6 Proportion of patients screened for delirium who had delirium. (PM - afternoon)

the introduction of the new process. Allowing the standardised design to be modified during testing also helped the dangle become acceptable to staff. A reminder in the form of a prompt on the daily goal sheet was also effective in driving process reliability, as it was a process already reliably delivered in our unit. The daily goals sheet is an

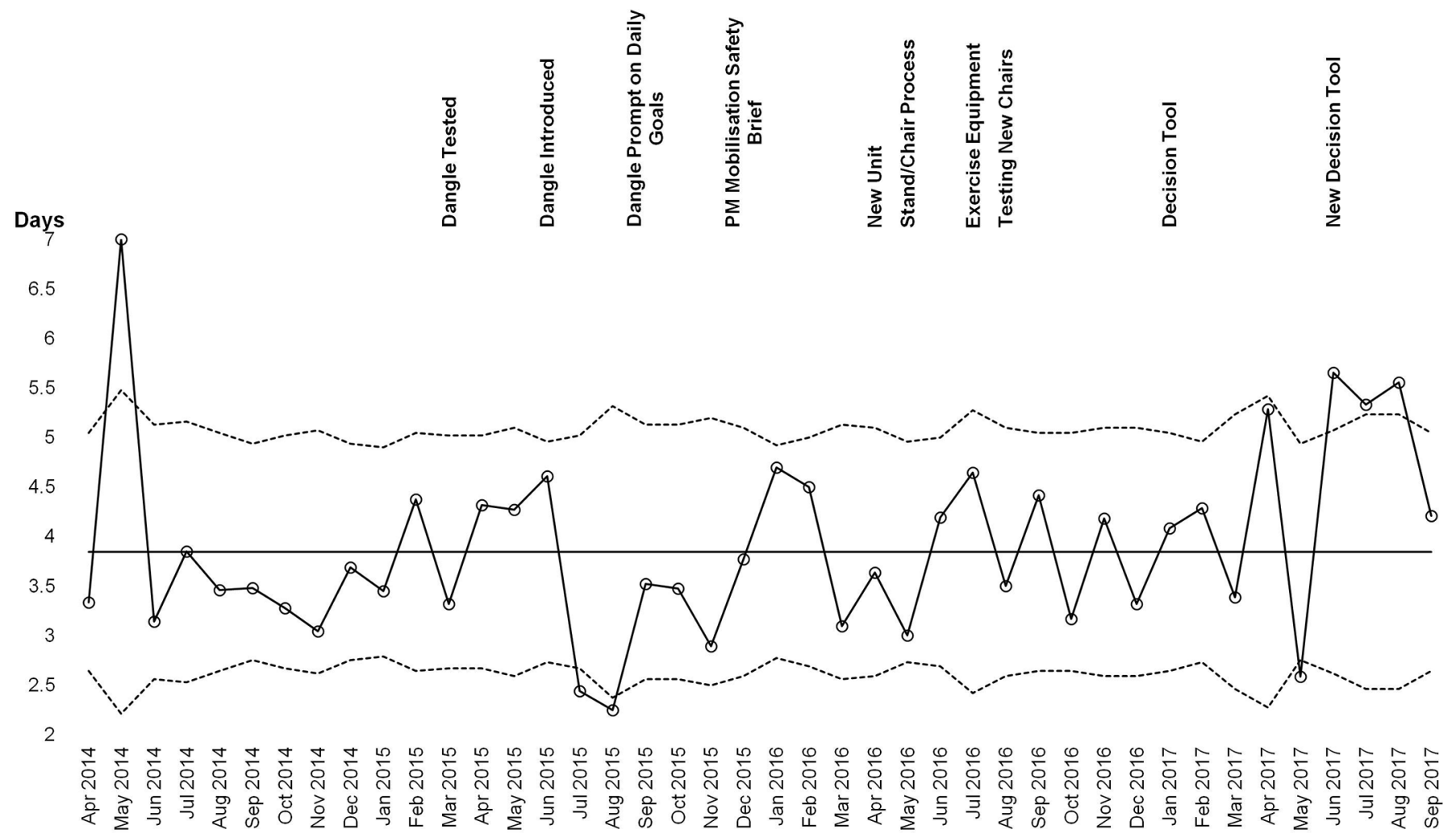

Figure $7 \quad U$ chart average length of stay on ventilator. PM, afternoon. 
example of a checklist used to ensure reminding the team to carry out key processes each day. However, adding a team brief in the early afternoon to plan mobilisation was not successful. We tried this as a form of redundancy, a second process to ensure that the first process was carried out, but as this was extra work, it proved to be very difficult to deliver.

The focus was the introduction of a process, based on published evidence suggesting a link to better outcomes. The design of our QI project is a before and after one, and so not designed for proving a causative relationship between process and outcome. We did measured delirium rate, average length of stay and average length of stay on a ventilator to see if outcomes changed in response to early mobilisation, and as part of a continuing long-term effort to improve outcomes in our patients. Delirium rates fell during the time of our project, but we cannot state with certainty that introducing early mobilisation was the cause, or an association. Delirium within ICU is known to be multifactorial, with immobility being one risk factor, and medication being another important risk factor, and we had tried other approaches to the prevention of delirium. The decrease was shortly after the introduction of the dangle process which suggests a link. This pattern is consistent with other studies ${ }^{19} 20$ which demonstrated that reduced sedation and mobilisation lead to reduced delirium rates. In these studies, the intervention was multifaceted and it is not possible to say what proportion of the effect size was due to mobilisation, if any. In our project and in these studies, it is possible that introduction of early mobilisation led to an unintended and unmeasured change in sedation practice. A limitation of our project is that we did not measure other variables which could affect the incidence of delirium, particularly sedative use or delirium management. It is biologically plausible that the change in delirium rate occurred through an unintended effect on sedative use. The sample size of our patients who met criteria for the dangle, and that of the patients who could be assessed for delirium were similar suggesting that the dangle was delivered to a large proportion of our patient population, and therefore could be an intervention at a large enough scale to affect, directly or indirectly, delirium rates. There were no other projects at the time specifically aimed at changing sedative use or affecting other risk factors for delirium. Although the mechanism is unknown, our data show that within our unit the introduction of the dangle is associated with change in outcome, and support the case for continued adoption of this process. We cannot be certain that continued use of the dangle ensures that delirium rates will not rise again, and efforts to prevent delirium will continue. Delirium rates fell further from January 2017 but this was felt to be due to a change in case mix as a consequence of the move from a standalone level 3 ICU to a combined level 2 and level 3 HDU and ICU.

Statistical process control chart analysis of the average length of stay shows special cause variation at the time of the introduction of the dangle process followed by reduced variability, suggesting a change to the system



Figure 8 Monthly proportion of patients who had a dangle, with delirium, and average length of stay. ALOS, average length of stay; ICU, intensive care unit. 
within our ICU (figures 5 and 8). The change in case mix after April 2016 was expected to affect average length of stay making analysis of the average length of stay more difficult. The link between mobilisation and improved muscle function was a rationale for the introduction of the dangle. We did not use any specific measure of muscle function, and therefore cannot say if any effect occurred. Average length of stay is affected by a number of variables including sedative use and discharge process from ICU, and so is not a specific measure of muscle function.

A reduction in length of stay has been found in only some studies. ${ }^{21}{ }^{22}$ It is the coincidence in time that supports a link between length of stay and mobilisation in our project. The special cause variation may be due to unmeasured variables. Variables that have been linked to optimal management of patients in the prevention of delirium and ICUAW along with early mobilisation include sedation practice, delirium management and spontaneous breathing trials. ${ }^{736}$ We had already achieved reliability with daily interruption of sedation and spontaneous breathing trials. Although there was no work at this time to specifically change other processes, we cannot say for certain that change did not occur and contribute to the pattern seen. It may be that the lack of sustained effect on length of stay indicates change in case mix, or that the dangle process is not the optimal one for our patients. There is no consensus in the literature on the optimal mobilisation process. A proportion of our patients went on to sit out of bed, stand or walk, but only if deemed safe to do so after a dangle (online supplementary figure 1 ). Therefore there was variation in the dose of mobilisation received. It is possible that if the dose was optimised, a clearer effect on outcomes would be seen. Patients selected for trials of mobilisation were expected to stay a significant amount of time on a ventilator. We applied the dangle to all patients who met the criteria in our project, and we may therefore have not seen the effect within subgroups of patients. A concern of staff initially was that patients might be mobilised too soon and this would cause physiological deterioration and 'set them back'. We cannot therefore say with confidence that we saw special cause variation in average length of stay as a consequence of the project, but can say that we did not see an increase which might be expected if there were adverse effects from mobilisation. In the same way, we did not see any increase in the average length of stay on the ventilator, our balancing measure. Measuring outcomes in our context considerably increases the confidence of staff in the adoption of a new and challenging process, which was the aim of the project. Having such outcome measures available provides useful data to support change, ensure that harm is avoided and is practical given the resources available within a clinical setting.

The involvement of a multiprofessional team, the adaptation of practices and guidelines for local use, the involvement of staff through focus groups in the clinical area, and iterative testing of change are strategies that are generalisable to other clinical areas and not specific to mobilisation. They represent a pragmatic and achievable approach to change. Standardisation and building reminders into current work processes are also generalisable and advocated by the Institute of Healthcare Improvement ${ }^{37}$ and the Scottish Patient Safety Programme ${ }^{38}$ Early mobilisation is relevant to all adult ICUs, although not necessarily to all patient groups. This represents our first attempt to bring rehabilitation for patients into critical care. The staff engagement approach has been important in the adoption of other rehabilitation processes such as the use of exercise equipment, special chairs, and latterly as standardised process for standing patients out of bed and caring for them in chairs instead of in bed. This staged approach has been important in making the introduction of care processes which initially seem difficult, and a radical departure from the norm, easier to introduce.

\section{FUTURE PLANS}

Our immediate plans are to ensure the sustainability of the dangle process. Ensuring delivery especially during busy periods is a challenge. We therefore plan to use the morning handover as an opportunity to plan ahead and deploy staff. We also plan to reintroduce the afternoon team brief as a safety brief as an opportunity for the team to organise mobilisation for patients still requiring a dangle. We are also introducing training for new staff.

We also plan to use the approach of standardising criteria and process for mobilisation to ensure that eligible patients stand or walk. Currently, patients stand or walk only if felt to be strong enough after a dangle. The impression of the project team is that more patients could be mobilised out of bed than is currently the case. This approach has also been adopted by a team in a surgical ward to test the introduction of standardised mobilisation and rehabilitation processes in postoperative patients.

Data to inform mobilisation practice, sedation practice, delirium and its management, and ventilation management are important to elucidate the optimal processes within intensive care. We will aim to develop further measures around sedation and delirium management at different stages of the patient journey, in addition to current measures. Ensuring consistent data collection in an NHS unit is a challenge, and the focus will be on data which support staff confidence in change.

\section{CONCLUSION}

A whole team approach to the adoption and adaptation of rehabilitation processes is effective in a general adult intensive care setting. A combination of strategies such as the use of short focus groups is pragmatic and makes the introduction of complex new processes easier to introduce. The use of improvement methodologies aimed at testing change and achieving reliability of processes is helpful for a team undertaking change. The effect of early mobilisation on delirium and other outcomes have been demonstrated elsewhere, but may be a useful approach 
in our context. The effect on these outcomes needs to be confirmed in other studies. Feedback from staff and patients is effective in building the confidence to adopt a change.

Acknowledgements The authors thank the entire staff group in the Monklands Hospital Intensive Care Unit for their enthusiasm, dedication and support throughout this project.

Contributors SC is the guarantor of the study and content. SC was responsible for the project design and data analysis. SC wrote and submitted the manuscript, and contributed to process redesign. SA was responsible for data collection, process redesign, preparing clinical documents and leading focus groups. LS was responsible for process redesign and leading staff focus groups.

Funding The authors have not declared a specific grant for this research from any funding agency in the public, commercial or not-for-profit sectors.

Competing interests None declared.

Provenance and peer review Not commissioned; externally peer reviewed.

Open access This is an open access article distributed in accordance with the Creative Commons Attribution Non Commercial (CC BY-NC 4.0) license, which permits others to distribute, remix, adapt, build upon this work non-commercially, and license their derivative works on different terms, provided the original work is properly cited, appropriate credit is given, any changes made indicated, and the use is non-commercial. See: http://creativecommons.org/licenses/by-nc/4.0/.

\section{REFERENCES}

1. Levels of critical care for adult patients. Intensive Care Society UK, 2009. https://www.ics.ac.uk/ICS/guidelines-and-standards.aspx

2. Ely EW, Inouye SK, Bernard GR, et al. Delirium in mechanically ventilated patients: validity and reliability of the confusion assessment method for the intensive care unit (CAM-ICU). JAMA 2001;286:2703-2010.

3. Delirium: prevention, diagnosis and management. National Institute of health and care excellence, 2010. https://www.nice.org.uk/ Guidance/CG103

4. Reade MC, Finfer S. Sedation and delirium in the intensive care unit. N Engl J Med 2014;370:444-54.

5. Bergeron N, Dubois MJ, Dumont M, et al. Intensive Care Delirium Screening Checklist: evaluation of a new screening tool. Intensive Care Med 2001;27:859-64.

6. Ely EW, Girard TD, Shintani AK, et al. Apolipoprotein E4 polymorphism as a genetic predisposition to delirium in critically ill patients. Crit Care Med 2007;35:112-7.

7. Barr J, Fraser GL, Puntillo K, et al. Clinical practice guidelines for the management of pain, agitation, and delirium in adult patients in the intensive care unit. Crit Care Med 2013;41:263-306.

8. Ouimet S, Kavanagh BP, Gottfried SB, et al. Incidence, risk factors and consequences of ICU delirium. Intensive Care Med 2007:33:66-73.

9. van den Boogaard M, Pickkers P, Slooter AJ, et al. Development and validation of PRE-DELIRIC (PREdiction of DELIRium in ICu patients) delirium prediction model for intensive care patients: observational multicentre study. BMJ 2012;344:e420.

10. Pisani MA, Kong SY, KasI SV, et al. Days of delirium are associated with 1-year mortality in an older intensive care unit population. $A m \mathrm{~J}$ Respir Crit Care Med 2009;180:1092-7.

11. Lin SM, Liu CY, Wang $\mathrm{CH}$, et al. The impact of delirium on the survival of mechanically ventilated patients. Crit Care Med 2004;32:2254-9.

12. van den Boogaard M, Schoonhoven L, Evers AW, et al. Delirium in critically ill patients: impact on long-term health-related quality of life and cognitive functioning. Crit Care Med 2012;40:112-8.

13. Ely EW, Shintani A, Truman B, et al. Delirium as a predictor of mortality in mechanically ventilated patients in the intensive care unit. JAMA 2004:291:1753-62.

14. Ely EW, Gautam S, Margolin R, et al. The impact of delirium in the intensive care unit on hospital length of stay. Intensive Care Med 2001;27:1892-900.
15. Stevens RD, Marshall SA, Cornblath DR, et al. A framework for diagnosing and classifying intensive care unit-acquired weakness. Crit Care Med 2009;37:S299-308.

16. De Jonghe B, Sharshar T, Lefaucheur JP, et al. Paresis acquired in the intensive care unit: a prospective multicenter study. JAMA 2002;288:2859-67.

17. Bednarík J, Vondracek P, Dusek L, et al. Risk factors for critical illness polyneuromyopathy. J Neurol 2005;252:343-51.

18. Herridge MS, Tansey CM, Matté A, et al. Functional disability 5 years after acute respiratory distress syndrome. $N$ Engl J Med 2011;364:1293-304.

19. Schweickert WD, Pohlman MC, Pohlman AS, et al. Early physical and occupational therapy in mechanically ventilated, critically ill patients: a randomised controlled trial. Lancet 2009;373:1874-82

20. Needham DM, Korupolu R, Zanni JM, et al. Early physical medicine and rehabilitation for patients with acute respiratory failure: a quality improvement project. Arch Phys Med Rehabil 2010;91:536-42.

21. Li Z, Peng X, Zhu B, et al. Active mobilization for mechanically ventilated patients: a systematic review. Arch Phys Med Rehabil 2013;94:551-61.

22. Tipping CJ, Harrold M, Holland A, et al. The effects of active mobilisation and rehabilitation in ICU on mortality and function: a systematic review. Intensive Care Med 2017:43:171-83.

23. Schaller SJ, Anstey M, Blobner M, et al. Early, goal-directed mobilisation in the surgical intensive care unit: a randomised controlled trial. Lancet 2016;388:1377-88.

24. Hodgson CL, Bailey M, Bellomo R, et al. A Binational Multicenter Pilot Feasibility Randomized Controlled Trial of Early Goal-Directed Mobilization in the ICU. Crit Care Med 2016;44:1145-52.

25. Rehabilitation after critical illness in adults. National Institute for Health and Care Excellence (NICE), 2009. https://www.nice.org.uk/ Guidance/CG832

26. Nydahl P, Sricharoencha T, Chandra S, et al. Safety of patient mobilisation and rehabilitation in the intensive care unit. Ann Am Thor Soc 2017:14:766-77.

27. Harrold ME, Salisbury LG, Webb SA, et al. Early mobilisation in intensive care units in Australia and Scotland: a prospective, observational cohort study examining mobilisation practises and barriers. Crit Care 2015;19:336.

28. Parry SM, Knight LD, Connolly B, et al. Factors influencing physical activity and rehabilitation in survivors of critical illness: a systematic review of quantitative and qualitative studies. Intensive Care Med 2017:43:531-42

29. Costa DK, White MR, Ginier E, et al. Identifying barriers to delivering the awakening and breathing coordination, delirium, and early exercise/mobility bundle to minimize adverse outcomes for mechanically ventilated patients: a systematic review. Chest 2017;152:304-11.

30. Audit of Critical Care in Scotland. The Scottish intensive care society audit group, 2017. www.sicsag.scot.nhs.uk/publications

31. Elliott SR. ICU delirium: a survey into nursing and medical staff knowledge of current practices and perceived barriers towards ICU delirium in the intensive care unit. Intensive Crit Care Nurs 2014;30:333-8

32. Bailey P, Thomsen GE, Spuhler VJ, et al. Early activity is feasible and safe in respiratory failure patients. Crit Care Med 2007;35:139-45.

33. Rogers EM. The Diffusion of innovations. 5th edn: Pub Simon and Schuster, 2003

34. The Health Foundation. Evidence Scan: What's getting in the way? Barriers to improvement in the NHS, 2015. http://www.health.org.uk/ publications

35. Resar RK. Making noncatastrophic health care processes reliable: Learning to walk before running in creating high-reliability organizations. Health Serv Res 2006;41:1677-89.

36. Nolan T, Resar R, Haraden C, et al. Improving the reliability of health care. ihi innovation series white paper. Boston: Institute for Healthcare Improvement, 2004.

37 .The Scottish Patient Safety Program. http://www.scottishpatients afetyprogramme.scot.nhs.uk

38. Pandharipande P, Banerjee A, McGrane S, et al. Liberation and animation for ventilated ICU patients: the ABCDE bundle for the back-end of critical care. Crit Care 2010;14:157. 